\title{
A PRELIMINARY CALIBRATION OF THE RR LYRAE PERIOD-LUMINOSITY RELATION AT MID-INFRARED WAVELENGTHS: WISE DATA
}

\author{
Barry F. Madore ${ }^{1}$, Douglas Hoffman ${ }^{2}$, Wendy L. Freedman ${ }^{1}$, Juna A. Kollmeier ${ }^{1}$, Andy Monson $^{1}$, \\ S. Eric Persson ${ }^{1}$, JefF A. Rich, Jr. ${ }^{1}$, Victoria Scowcroft ${ }^{1}$, And Mark Seibert ${ }^{1}$ \\ ${ }^{1}$ Observatories of the Carnegie Institution of Washington 813 Santa Barbara Street, Pasadena, CA 91101; barry@ obs.carnegiescience.edu, \\ wendy@obs.carnegiescience.edu, jak@obs.carnegiescience.edu, amonson@obs.carnegiescience.edu, persson@obs.carnegiescience.edu, \\ jrich@obs.carnegiescience.edu,vs@obs.carnegiescience.edu,mseibert@obs.carnegiescience.edu \\ ${ }^{2}$ Infrared Processing and Analysis Center 770 South Wilson, Pasadena, CA 91125 \\ Received 2013 March 3; accepted 2013 August 14; published 2013 October 7
}

\begin{abstract}

$$
\begin{aligned}
& M_{[W 1]}=-2.44( \pm 0.95) \times \log (P)-1.26( \pm 0.25) \sigma=0.10 \\
& M_{[W 2]}=-2.55( \pm 0.89) \times \log (P)-1.29( \pm 0.23) \sigma=0.10
\end{aligned}
$$$$
M_{[W 3]}=-2.58( \pm 0.97) \times \log (P)-1.32( \pm 0.25) \sigma=0.10 \text {. }
$$

Using time-resolved, mid-infrared data from the Wide-field Infrared Survey Explorer (WISE) and geometric parallaxes from the Hubble Space Telescope for four Galactic RR Lyrae variables, we derive the following Population II period-luminosity (PL) relations for the WISE [W1], [W2], and [W3] bands at 3.4, 4.6, and $12 \mu \mathrm{m}$, respectively:

The slopes and the scatter around the fits are consistent with a smooth extrapolation of those same quantities from previously published $K$-band observations at $2.2 \mu \mathrm{m}$, where the asymptotic (long-wavelength) behavior is consistent with a period-radius relation with a slope of 0.5 . No obvious correlation with metallicity (spanning 0.4 dex in $[\mathrm{Fe} / \mathrm{H}])$ is found in the residuals of the four calibrating RR Lyrae stars about the mean PL regression line.

Key words: stars: Population II - stars: variables: RR Lyrae

Online-only material: color figures

\section{INTRODUCTION}

The advantages of moving the Population I calibration of the Classical Cepheid period-luminosity (PL) relation (the Leavitt Law) from the optical to the infrared were outlined some three decades ago by McGonegal et al. (1982), and they have been borne out repeatedly over the years, as reviewed and elaborated upon by Freedman \& Madore (2010). But only now is it possible to extend these same advantages to the parallel path offered by the Population II variable stars (the short-period RR Lyrae variables and their longer-period siblings, the W Virginis stars). Two impressive accomplishments have made this possible: (1) the completion of the Wide-field Infrared Survey Explorer (WISE) mission (Wright et al. 2010) and the release of its sky survey of point sources measured in the mid-infrared (mid-IR) in four bands ranging from 3.4 to $22 \mu \mathrm{m}$, and then (2) the innovative application of the Hubble Space Telescope (HST) Fine-guidance Sensor (FGS) cameras to the determination of trigonometric parallaxes to four field RR Lyrae variables by Benedict et al. (2011).

The many, now well-known, advantages of calibrating and using PL relations in the mid-IR include the following: (1) the effects of line-of-sight extinction are reduced with respect to optical observations by at least an order of magnitude for even the shortest wavelength ([W1] at $3.4 \mu \mathrm{m})$ observations, (2) the concerns about the systematic impact of the possible non-universality of the reddening law are similarly reduced by going away from the optical and into the mid-IR, (3) the total amplitude of the light variation of the target star during its pulsation cycle is greatly reduced because of the largely diminished contribution of temperature variation to the change in surface brightness, in comparison to the much smaller (but essentially irreducible) wavelength-independent radius/areal variations, (4) the corresponding collapse in the width (i.e., intrinsic scatter) of the PL relations, again because of the reduced sensitivity of infrared luminosities to temperature variations (across the instability strip), combined with the intrinsic narrowness of the residual period-radius relations, and finally, (5) at mid-IR wavelengths, for the temperatures and surface gravities encountered in Population I and II Cepheids and RR Lyrae stars, there are so few metallic line or molecular transitions in those parts of the spectrum that atmospheric metallicity effects are expected to have minimal impact on the calibration. ${ }^{3}$ This is especially true for the RR Lyrae stars that are significantly hotter than their longer-period (cooler) Cepheid counterparts.

As described in Freedman et al. (2012), the Carnegie Hubble Program is designed to minimize and/or eliminate the remaining known systematics in the measurement of the Hubble constant using mid-IR data from NASA's Spitzer Space Telescope. Here we broaden the base in two distinct ways: (1) the incorporation of WISE mid-IR data and (2) the preliminary calibration of the Population II RR Lyrae variables as mid-IR distance indicators. This new initiative is known as the Carnegie RR Lyrae Program.

\section{THE CALIBRATORS: WISE OBSERVATIONS}

WISE conducted an all-sky survey at four mid-IR wavelengths, 3.4, 4.6, 12, and $22 \mu \mathrm{m}$ (hereafter $W 1, W 2, W 3$, and $W 4$, respectively). As such, all of the RR Lyrae variables with trigonometric parallaxes (Benedict et al. 2011) were also observed by the satellite. By design, the slowly precessing orbit of

\footnotetext{
3 One significant exception at $4.5 \mu \mathrm{m}$ has been noted for long-period Cepheids, where a $\mathrm{CO}$ molecular bandhead appears at temperatures below 4000 K; see Marengo et al. (2010), Scowcroft et al. (2011), and Monson et al. (2012); however, the temperatures of the RR Lyrae variables are so high in comparison to the long-period (classical) Cepheids, where this effect was discovered, that we expect no contribution of $\mathrm{CO}$ to the light or color curves of the RR Lyrae variables studied here. The W Virginis stars may be affected, and still need to be examined.
} 
Table 1

Mid-infrared (WISE) Magnitudes for Galactic RR Lyrae Variables

\begin{tabular}{lcclc}
\hline \hline Name & XZ Cyg & UV Oct & RR Lyr & SU Dra \\
\hline $\log (P)$ & -0.33107 & -0.26552 & -0.24655 & -0.18018 \\
Parallax (mas) & $1.67 \pm 0.17$ & $1.71 \pm 0.10$ & $3.77 \pm 0.13$ & $1.42 \pm 0.16$ \\
$A_{V}{ }^{\mathrm{a}}$ & 0.30 & 0.28 & 0.13 & 0.03 \\
$A_{W 1}$ & 0.020 & 0.018 & 0.008 & 0.002 \\
$A_{W 2}$ & 0.016 & 0.015 & 0.007 & 0.002 \\
$A_{W 3}$ & 0.002 & 0.001 & 0.001 & 0.000 \\
LKH Corr. & -0.09 & -0.03 & -0.02 & -0.11 \\
$(m-M)_{o}$ & 8.98 & 8.87 & 7.14 & 9.35 \\
$m[W 1]$ & 8.610 & 8.156 & 6.469 & 8.584 \\
$\sigma[W 1]$ & 0.010 & 0.017 & 0.017 & 0.019 \\
Ampl $[W 1]$ & 0.21 & 0.24 & 0.24 & 0.29 \\
$m[W 2]$ & 8.616 & 8.172 & 6.456 & 8.580 \\
$\sigma[W 2]$ & 0.010 & 0.014 & 0.017 & 0.019 \\
Ampl [W2] & 0.20 & 0.21 & 0.27 & 0.29 \\
$m[W 3]$ & 8.579 & 8.110 & 6.460 & 8.543 \\
$\sigma[W 3]$ & 0.010 & 0.016 & 0.015 & 0.018 \\
Ampl $[W 3]$ & 0.19 & 0.22 & 0.24 & 0.27 \\
$M[W 1]$ & $-0.39(0.23)$ & $-0.73(0.13)$ & $-0.68(0.08)$ & $-0.77(0.26)$ \\
$M[W 2]$ & $-0.38(0.23)$ & $-0.71(0.13)$ & $-0.69(0.08)$ & $-0.77(0.26)$ \\
$M[W 3]$ & $-0.40(0.23)$ & $-0.76(0.13)$ & $-0.68(0.08)$ & $-0.81(0.26)$ \\
\hline & & & &
\end{tabular}

Note. ${ }^{\text {a }}$ Benedict et al. (2011), where $A_{\text {band }} / E(B-V)=0.20,0.16,0.03$ for $W 1, W 2$, and $W 3$, respectively.

WISE allowed the satellite to scan across every object on the sky at least 12 times (with progressively more coverage at higher ecliptic latitudes). These successive observations were obtained within a relatively narrow window of time (over about $18 \mathrm{hr}$ for those fields nearest the ecliptic equator) with each observation being separated by about 90 minutes (the orbital period of the satellite). RR Lyrae stars have periods that are generally less than $16 \mathrm{hr}$, meaning that even the sparsest of these multiple mid-IR observations covered at least one full pulsational cycle of these particular variable stars.

The light curves based on the time-resolved WISE observations of our calibrating stars (Tables 1-5) are shown in Figures 1 and 2 for the four RR Lyrae variables, SU Dra, $R R L y r, X Z C y g$, and UV Oct. These stars were observed by WISE 51, 23, 24, and 23 times, respectively. Data were retrieved from the WISE All-Sky Single Exposure (L1b) Source Table, which is available at the Infrared Science Archive (http://irsa.ipac.caltech.edu/Missions/wise.html). The source positions were queried with a 2.5 arcsec cone search radius, ignoring observations flagged as contaminated by artifacts. The observations are very uniformly distributed around the cycle and the resulting light curves are exceedingly well defined. All three of the shortest-wavelength light curves show convergence in their properties, exemplified by their mutual phases, shapes, and amplitudes. ${ }^{4}$ As expected these light curves closely track the anticipated light variations due to surface-area variations of the star, where at these wavelengths the sensitivity of the surface brightness to a temperature variation is much diminished as compared to its sensitivity at optical wavelengths. This then fully accounts for the mutual phasing (tracking the radius variations and not the off-set temperature variations), the shape (the

\footnotetext{
4 It should be noted that RR Lyr is saturated in the WISE data, and there is a documented flux over-estimation bias for saturated sources, especially in $W 2$ (Cutri et al. 2012). However, we have made no correction for the bias as it only becomes detectable for sources brighter than 4.0 mag for $W 1$ and 6.0 mag for $W 2$.
}

Table 2

WISE Observations of RR Lyrae

\begin{tabular}{|c|c|c|c|c|c|c|}
\hline $\operatorname{MJD}(2,400,000 .+)$ & $3.4 \mu \mathrm{m}$ & Error & $4.6 \mu \mathrm{m}$ & Error & $12 \mu \mathrm{m}$ & Error \\
\hline 55312.047509 & 6.612 & 0.019 & 6.448 & 0.022 & 6.490 & 0.014 \\
\hline 55312.179813 & 6.542 & 0.034 & 6.554 & 0.019 & 6.595 & 0.019 \\
\hline 55312.312118 & 6.387 & 0.031 & 6.488 & 0.020 & 6.428 & 0.013 \\
\hline 55312.378206 & 6.358 & 0.049 & 6.349 & 0.021 & 6.428 & 0.019 \\
\hline 55312.444422 & 6.317 & 0.030 & 6.390 & 0.019 & 6.395 & 0.014 \\
\hline 55312.510510 & 6.314 & 0.032 & 6.376 & 0.018 & 6.420 & 0.019 \\
\hline 55312.576726 & 6.356 & 0.034 & 6.466 & 0.017 & 6.416 & 0.016 \\
\hline 55312.642814 & 6.476 & 0.031 & 6.450 & 0.021 & 6.514 & 0.016 \\
\hline 55312.709030 & 6.579 & 0.026 & 6.496 & 0.021 & 6.528 & 0.016 \\
\hline 55312.775119 & 6.580 & 0.041 & 6.604 & 0.019 & 6.643 & 0.017 \\
\hline 55312.775246 & 6.543 & 0.029 & 6.629 & 0.020 & 6.630 & 0.020 \\
\hline 55312.841334 & 6.506 & 0.028 & 6.531 & 0.019 & 6.471 & 0.018 \\
\hline 55312.907423 & 6.423 & 0.032 & 6.400 & 0.016 & 6.396 & 0.013 \\
\hline 55312.973639 & 6.518 & 0.04 & 6.395 & 0.018 & 6.372 & 0.017 \\
\hline 55313.039855 & 6.323 & 0.037 & 6.394 & 0.020 & 6.378 & 0.018 \\
\hline 55313.105943 & 6.716 & 0.04 & 6.332 & 0.017 & 6.384 & 0.019 \\
\hline 55313.238247 & 6.589 & 0.030 & 6.561 & 0.017 & 6.495 & 0.016 \\
\hline 55313.304463 & 6.587 & 0.029 & 6.571 & 0.015 & 6.506 & 0.016 \\
\hline 55313.436767 & 6.437 & 0.044 & 6.390 & 0.018 & 6.387 & 0.014 \\
\hline 55313.502856 & 6.390 & 0.037 & 6.366 & 0.013 & 6.392 & 0.014 \\
\hline 55313.569072 & 6.284 & 0.034 & 6.335 & 0.020 & 6.371 & 0.016 \\
\hline 55313.701376 & 6.442 & 0.030 & 6.433 & 0.028 & 6.422 & 0.014 \\
\hline 55313.833680 & 6.542 & 0.032 & 6.491 & 0.022 & 6.523 & 0.017 \\
\hline 55321.904496 & 6.451 & 0.038 & 6.513 & 0.018 & 6.463 & 0.016 \\
\hline
\end{tabular}

Table 3

WISE Observations of SU Draconis

\begin{tabular}{lcccccc}
\hline \hline $\mathrm{MJD}(2,400,000 .+)$ & $3.4 \mu \mathrm{m}$ & Error & $4.6 \mu \mathrm{m}$ & Error & $12 \mu \mathrm{m}$ & Error \\
\hline 55315.013213 & 8.561 & 0.019 & 8.502 & 0.017 & 8.507 & 0.037 \\
55315.145517 & 8.501 & 0.021 & 8.474 & 0.022 & 8.470 & 0.043 \\
55315.145644 & 8.477 & 0.022 & 8.479 & 0.021 & 8.404 & 0.039 \\
55315.277821 & 8.544 & 0.023 & 8.523 & 0.019 & 8.482 & 0.040 \\
55315.277949 & 8.532 & 0.022 & 8.473 & 0.023 & 8.495 & 0.040 \\
55315.344037 & 8.601 & 0.017 & 8.581 & 0.013 & 8.568 & 0.040 \\
55315.410125 & 8.631 & 0.022 & 8.628 & 0.019 & 8.570 & 0.044 \\
55315.410253 & 8.602 & 0.021 & 8.588 & 0.017 & 8.589 & 0.046 \\
55315.476341 & 8.697 & 0.020 & 8.696 & 0.017 & 8.684 & 0.046 \\
55315.542559 & 8.792 & 0.021 & 8.809 & 0.017 & 8.776 & 0.052 \\
55315.608647 & 8.631 & 0.017 & 8.646 & 0.019 & $\ldots$ & $\ldots$ \\
55315.674863 & 8.522 & 0.021 & 8.513 & 0.016 & 8.464 & 0.043 \\
55315.740952 & 8.498 & 0.017 & 8.481 & 0.017 & 8.449 & 0.043 \\
55315.807167 & 8.484 & 0.025 & 8.484 & 0.020 & 8.418 & 0.037 \\
55315.873256 & 8.501 & 0.020 & 8.514 & 0.019 & 8.508 & 0.039 \\
55315.939472 & 8.541 & 0.020 & 8.513 & 0.020 & 8.478 & 0.040 \\
55316.005560 & 8.554 & 0.018 & 8.571 & 0.016 & 8.511 & 0.040 \\
55316.071776 & 8.608 & 0.021 & 8.620 & 0.019 & 8.608 & 0.045 \\
55316.137864 & 8.689 & 0.021 & 8.635 & 0.019 & 8.638 & 0.043 \\
55316.204080 & 8.787 & 0.018 & 8.772 & 0.024 & 8.677 & 0.043 \\
55316.270169 & 8.607 & 0.016 & 8.655 & 0.018 & 8.575 & 0.042 \\
55316.402473 & 8.467 & 0.019 & 8.520 & 0.022 & 8.509 & 0.045 \\
55316.534777 & 8.492 & 0.026 & 8.513 & 0.020 & 8.448 & 0.039 \\
\hline
\end{tabular}

cycloid-like radius variation, in contrast to the highly asymmetric color/temperature variation), and the low amplitude (around 0.3 mag, peak-to-peak, in line with the small, radius-induced cyclical change in surface area of these stars).

The non-parametric fitting methodology, GLOESS, was used to derive intensity-averaged magnitudes and amplitudes, as given in Table 1 (see Persson et al. 2004 for a description and an early application of this fitting technique). 
Table 4

WISE Observations of UV Oct

\begin{tabular}{lcccccc}
\hline \hline MJD(2,400,000.+) & $3.4 \mu \mathrm{m}$ & Error & $4.6 \mu \mathrm{m}$ & Error & $12 \mu \mathrm{m}$ & Error \\
\hline 55270.919633 & 8.082 & 0.025 & 8.114 & 0.020 & 8.063 & 0.031 \\
55271.052065 & 8.091 & 0.021 & 8.089 & 0.015 & 7.985 & 0.029 \\
55271.184369 & 8.151 & 0.019 & 8.194 & 0.020 & 8.147 & 0.032 \\
55271.316673 & 8.314 & 0.016 & 8.355 & 0.018 & 8.245 & 0.032 \\
55271.382761 & 8.173 & 0.028 & 8.162 & 0.019 & 8.198 & 0.032 \\
55271.448977 & 8.065 & 0.019 & 8.112 & 0.017 & 8.045 & 0.030 \\
55271.515192 & 8.099 & 0.020 & 8.076 & 0.016 & 8.051 & 0.029 \\
55271.581281 & 8.065 & 0.018 & 8.096 & 0.016 & $\ldots$ & $\ldots$ \\
55271.647496 & 8.141 & 0.019 & 8.108 & 0.021 & 8.063 & 0.030 \\
55271.713585 & 8.151 & 0.022 & 8.198 & 0.019 & 8.119 & 0.034 \\
55271.779800 & 8.191 & 0.020 & 8.192 & 0.022 & 8.165 & 0.030 \\
55271.845888 & 8.300 & 0.022 & 8.299 & 0.020 & 8.243 & 0.034 \\
55271.846016 & 8.295 & 0.022 & 8.277 & 0.022 & 8.250 & 0.032 \\
55271.912104 & 8.231 & 0.019 & 8.234 & 0.017 & 8.191 & 0.032 \\
55271.978320 & 8.076 & 0.026 & 8.111 & 0.016 & 8.062 & 0.028 \\
55272.044408 & 8.046 & 0.018 & 8.149 & 0.017 & 8.029 & 0.029 \\
55272.176712 & 8.071 & 0.021 & 8.098 & 0.019 & 8.070 & 0.033 \\
55272.242928 & 8.151 & 0.017 & 8.132 & 0.013 & 8.060 & 0.029 \\
55272.309016 & 8.201 & 0.022 & 8.204 & 0.019 & 8.148 & 0.033 \\
55272.309144 & 8.189 & 0.022 & 8.215 & 0.022 & 8.146 & 0.031 \\
55272.441448 & 8.321 & 0.021 & 8.324 & 0.020 & 8.203 & 0.033 \\
55272.573751 & 8.062 & 0.023 & 8.144 & 0.028 & 8.000 & 0.030 \\
55272.706056 & 8.121 & 0.017 & 8.140 & 0.013 & 8.074 & 0.029 \\
\hline & & & & & &
\end{tabular}

\section{RR LYRAE PERIOD-LUMINOSITY RELATIONS}

Table 1 contains the parameters needed to compute absolute mean magnitudes. The parallaxes, $E(B-V)$ reddenings, and Lutz-Kelker-Hanson (LKH; Lutz \& Kelker 1973; Hanson 1979) corrections as taken from Benedict et al. (2011), are listed for convenience. We have converted the $A_{V}$ extinctions listed by Benedict et al. (2011) to those in the $W 1$ and $W 2$ bands using the Yuan et al. (2013) compilation of $A_{\mathrm{WISE}} / E(B-V)$ results with $A_{V} / E(B-V)=3$.1. Indebetouw et al. (2005) give values of $A_{\text {Spitzer }} / A_{K},{ }^{5}$ which we converted to $A_{\mathrm{WISE}} / A_{V}$ via the Cardelli et al. (1989) law. The values of $A_{\mathrm{WISE}} / A_{V}$ given by Yuan et al. (2013) and our pseudo-values from Indebetouw et al. (2005) agree well. Neither the Yuan et al. (2013) nor Indebetouw et al. (2005) results extend to the $W 3$ band, and here we have referred to Fitzpatrick (1999) for an approximate value. The extinctions for the four stars are so small that they make no difference to the absolute magnitude values and we take $A_{W 3} / A_{V}$ to be 0.01 . The mid-IR extinctions $A_{\text {WISE }} / A_{V}$ we adopt are $0.065,0.052$, and 0.01 for $W 1, W 2$, and $W 3$, respectively. The adopted mid-IR extinctions $A_{\mathrm{WISE}} / E(B-V)$ are also given in Table 1 . The above parameters and our observed mean magnitudes lead to the $W 1, W 2$, and $W 3$ absolute magnitudes in Table $1 .^{6}$ The PL relations for RR Lyrae variables follow:

$$
\begin{gathered}
M_{[W 1]}=-2.44( \pm 0.95) \times \log (P)-1.26( \pm 0.25) \sigma=0.10 \\
M_{[W 2]}=-2.55( \pm 0.89) \times \log (P)-1.29( \pm 0.23) \sigma=0.10 \\
M_{[W 3]}=-2.58( \pm 0.97) \times \log (P)-1.32( \pm 0.25) \sigma=0.10 .
\end{gathered}
$$

The absolute magnitude values and the respective fits to the first two WISE bands as well as $K$-band data (from Benedict

\footnotetext{
The Indebetouw et al. values, strictly speaking, actually apply to the Spitzer channels 1 and 2 bands which are quite close to the $W 1$ and $W 2$ bands; the differences can be safely ignored.

6 Our magnitudes differ slightly from those in Benedict et al. (2011). These differences stem from our use of different reddening law assumptions together with typographical errors in their Table 8 (G. F. Benedict 2013, private communication).
}

\begin{tabular}{|c|c|c|c|c|c|c|}
\hline $\operatorname{MJD}(2,400,000 .+)$ & $3.4 \mu \mathrm{m}$ & Error & $4.6 \mu \mathrm{m}$ & Error & $12 \mu \mathrm{m}$ & Error \\
\hline 55334.008212 & 8.589 & 0.016 & 8.586 & 0.017 & 8.548 & 0.037 \\
\hline 55334.140516 & 8.635 & 0.016 & 8.633 & 0.017 & 8.584 & 0.040 \\
\hline 55334.272820 & 8.748 & 0.019 & 8.757 & 0.017 & 8.687 & 0.048 \\
\hline 55334.339035 & 8.614 & 0.018 & 8.637 & 0.021 & 8.680 & 0.049 \\
\hline 55334.405124 & 8.555 & 0.019 & 8.577 & 0.018 & 8.509 & 0.041 \\
\hline 55334.471212 & 8.552 & 0.028 & 8.508 & 0.020 & 8.519 & 0.039 \\
\hline 55334.471339 & 8.532 & 0.018 & 8.490 & 0.021 & 8.540 & 0.048 \\
\hline 55334.537428 & 8.554 & 0.019 & 8.654 & 0.020 & 8.550 & 0.040 \\
\hline 55334.603643 & 8.528 & 0.025 & 8.536 & 0.015 & 8.642 & 0.040 \\
\hline 55334.669732 & 8.678 & 0.020 & 8.699 & 0.020 & 8.667 & 0.046 \\
\hline 55334.735820 & 8.777 & 0.023 & 8.725 & 0.021 & 8.738 & 0.044 \\
\hline 55334.735947 & 8.667 & 0.023 & 8.698 & 0.015 & 8.812 & 0.046 \\
\hline 55334.868124 & 8.536 & 0.023 & 8.553 & 0.022 & 8.536 & 0.038 \\
\hline 55334.868251 & 8.488 & 0.026 & 8.519 & 0.015 & 8.597 & 0.045 \\
\hline 55334.934340 & 8.553 & 0.019 & 8.541 & 0.017 & 8.546 & 0.044 \\
\hline 55335.000428 & 8.533 & 0.027 & 8.556 & 0.020 & 8.392 & 0.035 \\
\hline 55335.000555 & 8.554 & 0.022 & 8.492 & 0.020 & 8.633 & 0.046 \\
\hline 55335.066644 & 8.593 & 0.019 & 8.612 & 0.018 & 8.586 & 0.047 \\
\hline 55335.132732 & 8.602 & 0.026 & 8.643 & 0.020 & 8.642 & 0.041 \\
\hline 55335.132859 & 8.597 & 0.019 & 8.581 & 0.018 & 8.709 & 0.047 \\
\hline 55335.198947 & 8.742 & 0.017 & 8.757 & 0.021 & 8.651 & 0.044 \\
\hline 55335.265036 & 8.627 & 0.021 & 8.669 & 0.016 & 8.591 & 0.039 \\
\hline 55335.265163 & 8.658 & 0.023 & 8.636 & 0.021 & 8.682 & 0.044 \\
\hline 55335.331251 & 8.552 & 0.017 & 8.601 & 0.021 & 8.461 & 0.041 \\
\hline 55335.397340 & 8.468 & 0.019 & 8.520 & 0.016 & 8.560 & 0.039 \\
\hline 55335.397467 & 8.514 & 0.024 & 8.543 & 0.020 & 8.574 & 0.044 \\
\hline 55335.463555 & 8.552 & 0.017 & 8.559 & 0.019 & 8.501 & 0.043 \\
\hline 55335.529644 & 8.587 & 0.019 & 8.588 & 0.016 & 8.619 & 0.040 \\
\hline 55335.529771 & 8.505 & 0.021 & 8.584 & 0.021 & 8.669 & 0.045 \\
\hline 55335.595859 & 8.650 & 0.017 & 8.688 & 0.020 & 8.639 & 0.047 \\
\hline 55335.661948 & 8.844 & 0.022 & 8.729 & 0.019 & 8.698 & 0.046 \\
\hline 55335.662075 & 8.648 & 0.021 & 8.699 & 0.023 & 8.751 & 0.050 \\
\hline 55335.728163 & 8.674 & 0.017 & 8.666 & 0.019 & 8.577 & 0.044 \\
\hline 55335.794252 & 8.623 & 0.023 & 8.521 & 0.019 & 8.459 & 0.040 \\
\hline 55335.794379 & 8.509 & 0.023 & 8.706 & 0.029 & 8.514 & 0.043 \\
\hline 55335.860467 & 8.502 & 0.018 & 8.562 & 0.017 & 8.441 & 0.040 \\
\hline 55335.926556 & 8.531 & 0.021 & 8.546 & 0.020 & 8.442 & 0.040 \\
\hline 55335.992771 & 8.617 & 0.019 & 8.604 & 0.017 & 8.512 & 0.040 \\
\hline 55336.058860 & 8.648 & 0.021 & 8.687 & 0.020 & 8.542 & 0.042 \\
\hline 55336.125075 & 8.743 & 0.020 & 8.781 & 0.026 & 8.626 & 0.041 \\
\hline 55336.191164 & 8.705 & 0.022 & 8.702 & 0.020 & 8.632 & 0.045 \\
\hline 55336.257379 & 8.610 & 0.019 & 8.593 & 0.024 & 8.439 & 0.036 \\
\hline 55336.323468 & 8.561 & 0.021 & 8.502 & 0.019 & 8.478 & 0.039 \\
\hline 55336.389683 & 8.554 & 0.024 & 8.580 & 0.026 & 8.427 & 0.038 \\
\hline 55336.455772 & 8.626 & 0.022 & 8.584 & 0.017 & 8.595 & 0.048 \\
\hline 55336.521985 & 8.685 & 0.017 & 8.712 & 0.014 & 8.589 & 0.039 \\
\hline 55336.588074 & 8.738 & 0.021 & 8.729 & 0.023 & 8.597 & 0.044 \\
\hline 55336.654289 & 8.776 & 0.018 & 8.781 & 0.014 & 8.564 & 0.040 \\
\hline 55336.720377 & 8.425 & 0.021 & 8.521 & 0.017 & 8.561 & 0.039 \\
\hline 55336.852681 & 8.518 & 0.018 & 8.499 & 0.016 & 8.481 & 0.038 \\
\hline 55336.984985 & 8.634 & 0.019 & 8.599 & 0.017 & 8.730 & 0.046 \\
\hline
\end{tabular}

Table 5

WISE Observations of XZ Cyg

et al. 2011 and Dall'Ora et al. 2004, respectively) are shown in Figure 3. Despite the very small (less than a factor of two) range in period covered by RR Lyrae stars, the PL relations are well defined, largely because of their intrinsically small scatter. The intrinsic scatter is especially well illustrated by the $K$-band PL relation, where we also show the RR Lyrae data of Dall'Ora et al. for 21 fundamental-mode RR Lyrae stars in the well-populated LMC globular cluster, Reticulum (shifted by $18.47 \mathrm{mag}$ ). A comparison of these two data sets is very illuminating. The slope of the adopted PL relation at $K$ and the total width of it, as defined by the two samples, are for 

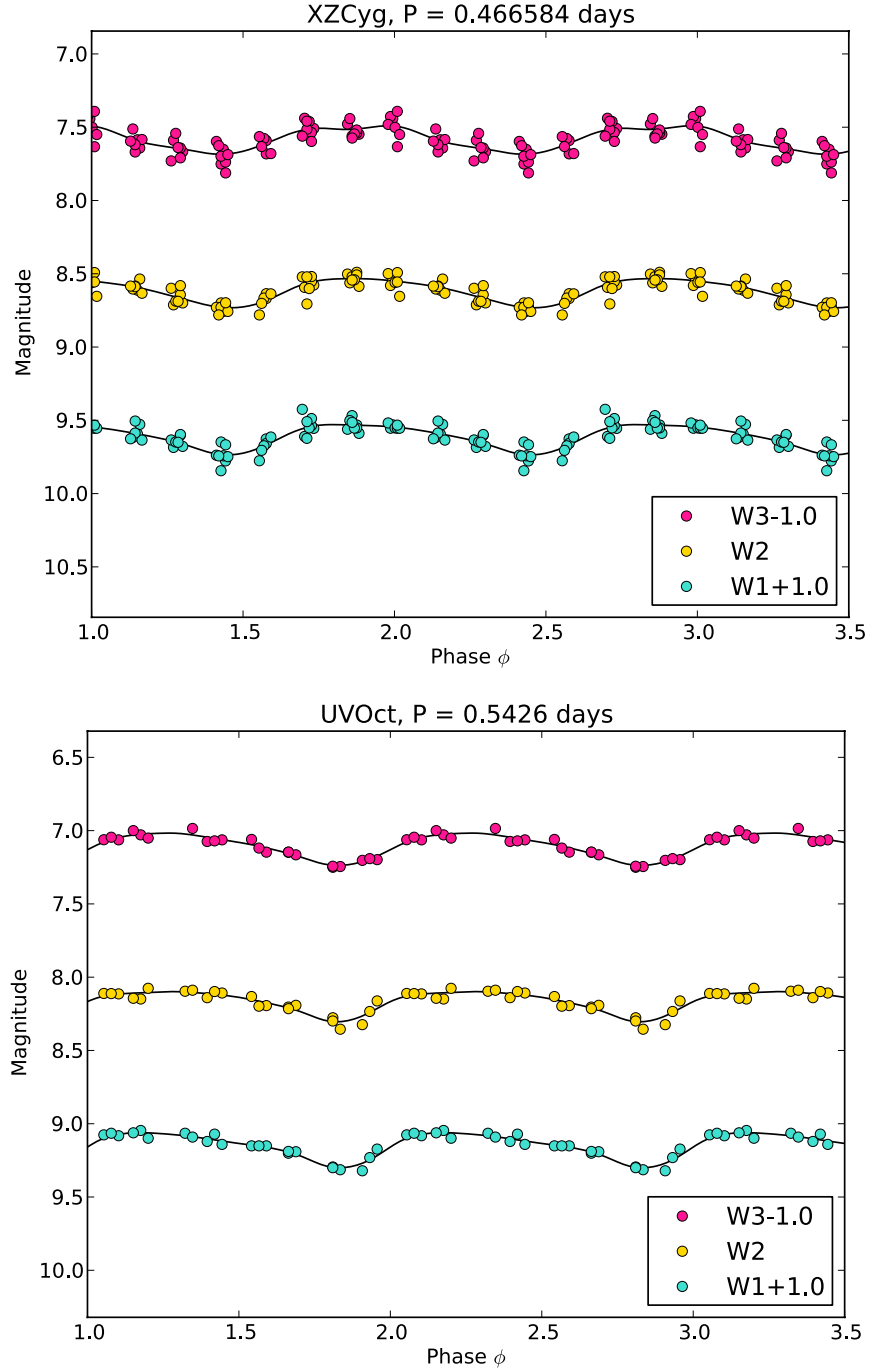

Figure 1. WISE mid-infrared light curves for XZ Cyg (upper panel) and UV Oct (lower panel) phase-folded over two and a half cycles using the periods given in the titles. GLOESS fits are shown as solid black lines.

(A color version of this figure is available in the online journal.)

all intents and purposes, identical. The very good agreement in these two independently determined slopes and the small dispersion in each of the data sets suggest that the means of the Milky Way variables are already well constrained even though the Galactic calibrating sample itself is currently very small.

On the other hand, we note that the small (observed) scatter of the Milky Way RR Lyrae variables around each of the adopted PL relations is apparently at variance with the individually quoted error bars for each of the calibrating variables. That is, the formal scatter of \pm 0.10 mag in the WISE PL relations is compared with the quoted parallax errors on the individual distance moduli of $\pm 0.22, \pm 0.16, \pm 0.25$, and $\pm 0.07 \mathrm{mag}$ for XZ Cyg, UV Oct, SU Dra, and RR Lyr, respectively. The average scatter for the variables $( \pm 0.18 \mathrm{mag})$ is then about two times larger than their observed scatter around the PL fit. This suggests that the published errors may be somewhat overestimated. There are independent data that support this assertion. The 10 Galactic Cepheids for which Benedict et al. (2011) obtained parallaxes, using the same instrument, telescope, and reduction methodology, have individually quoted internal errors in their true distance moduli ranging from \pm 0.11 to $\pm 0.30 \mathrm{mag}$. Their
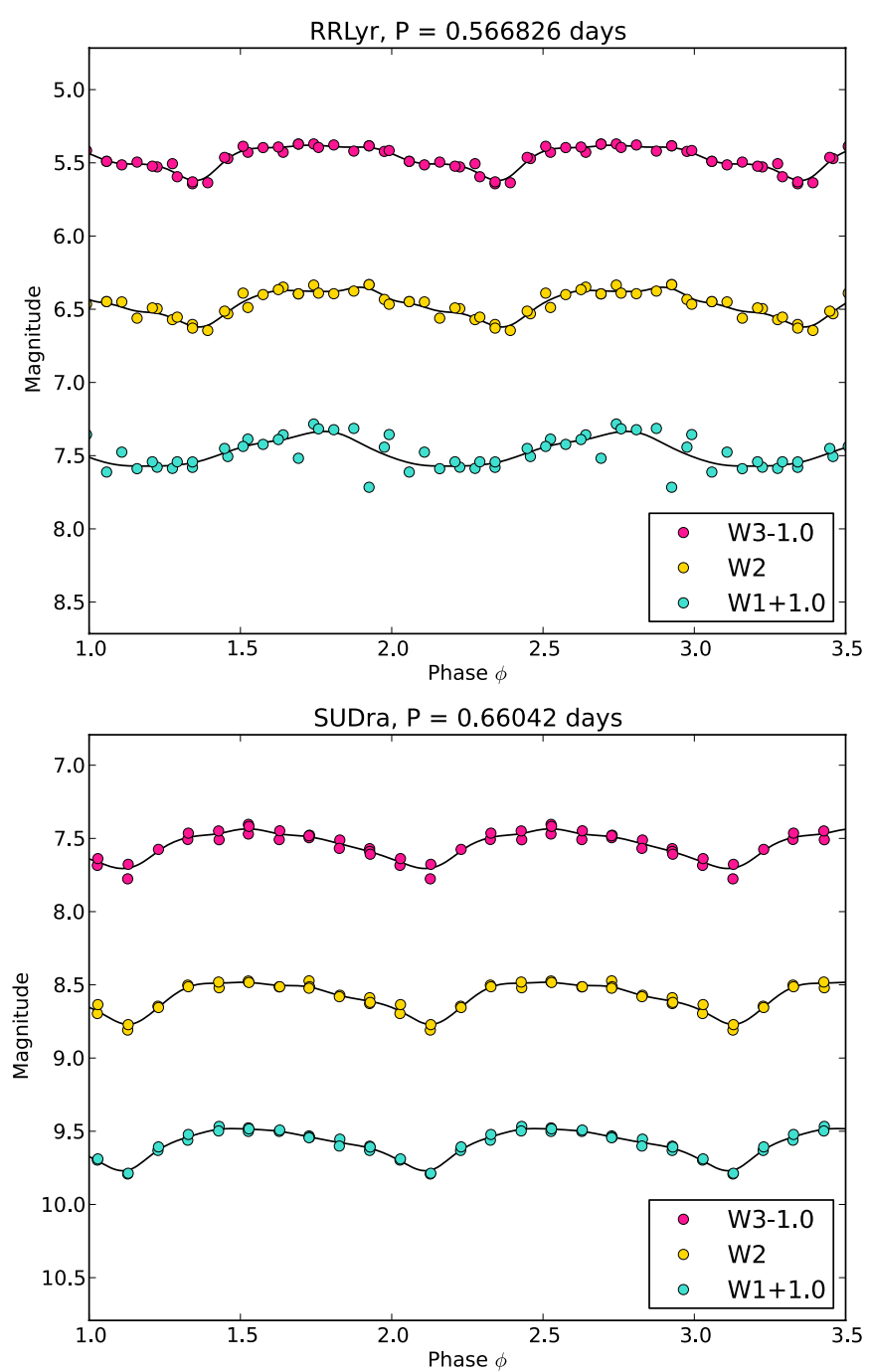

Figure 2. WISE mid-infrared light curves for RR Lyr (upper panel) and SU Dra (lower panel) phase-folded over two and a half cycles using the periods given in the titles. GLOESS fits are shown as solid black lines.

(A color version of this figure is available in the online journal.)

average uncertainty is $\pm 0.19 \mathrm{mag}$, and yet once again, as with the RR Lyrae variables, the PL fit to these data yields a formal dispersion of only $\pm 0.10 \mathrm{mag}$. In both cases the observed dispersions for the Galactic samples, are in total agreement with independently determined dispersions for the much more robustly determined dispersions for the LMC samples. We suggest therefore that the random errors reported for the HST parallaxes for both the Cepheids and the RR Lyrae variables may have been over-estimated. This is not simply of academic interest. If the observed scatter is used to calculate the systematic uncertainty in the calibration of the RR Lyrae PL relation, that uncertainty would be $0.10 / \sqrt{4}= \pm 0.05 \mathrm{mag}$, a $2 \%-3 \%$ error in the Population II distance scale. However, if the quoted errors on the individual distance moduli are used, then the uncertainty rises to $0.18 / \sqrt{4}= \pm 0.09 \mathrm{mag}$, a $5 \%$ error. Similar conclusions would also apply to the base uncertainty in the Cepheid distance scale using the Benedict sample; is the uncertainty in the Galactic Cepheid zero point $1.6 \%$ in distance, or is it $3.0 \%$ ? It is therefore important to note that in their first paper discussing the use of FGS on HST, Benedict et al. (2002) state that the "standard deviations of the HST and Hipparcos data points may have been 


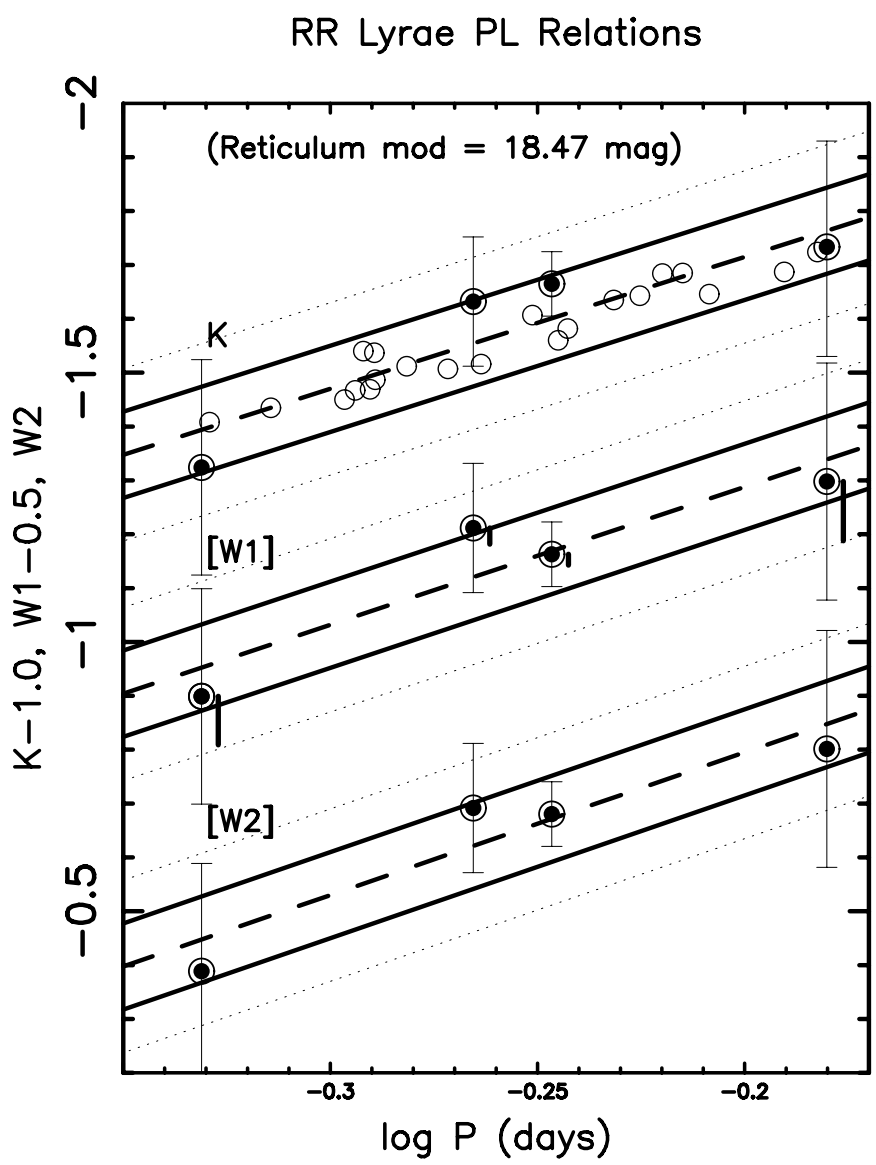

Figure 3. RR Lyrae PL relations in the $K$ band (top) and the two WISE bands [W1] (middle) and [W2] (bottom). The $K$-band relation also contains data from Dall'Ora et al. (2004) for the LMC globular cluster, Reticulum, shifted by $18.47 \mathrm{mag}$. (This distance modulus shift is remarkably close to the independently determined true modulus of $18.48 \mathrm{mag}$ recently reported by Monson et al. 2012 for the LMC Cepheid mid-infrared distance modulus.) The Reticulum data are shown only for the RRab (fundamental) pulsators, and are presented here to illustrate that they are consistent in slope and scatter in comparison with the Galactic calibration. A detailed discussion of Reticulum will be given in a forthcoming paper (Monson et al. 2012). The solid lines flanking each of the fitted PL relations are each separated by two sigma from their respective ridge lines. Despite the small numbers of stars represented here, the full width of the PL relation in each of the bands is well defined. The solid vertical lines to the right of each of the [W1] data points represents the LKH correction applied by Benedict et al. (2011).

overstated by a factor of $\sim 1.5$ " and since the Hipparcos errors had been subjected to many confirming tests "it is likely that the HST errors are overstated." Parallaxes from Gaia are anxiously awaited because they will improve the number of calibrators by orders of magnitude and convincingly set the zero point.

In Figure 3 we show, using thick vertical lines, the full magnitude of the LKH corrections as published by Benedict et al. (2011) and applied to the true distance moduli used here. It is noteworthy that, if these corrections had not been applied, the dispersion in the data points around the fit would have exceeded the independently determined dispersion from the Reticulum data, and the slope of the Milky Way solution would have been more shallow than the LMC slope. We take the final agreement of both the slopes and the dispersions to suggest that the individually determined and independently applied LKH corrections are appropriate.

Finally, it needs to be noted that Klein et al. (2011) have published slopes that are much shallower than the ones derived

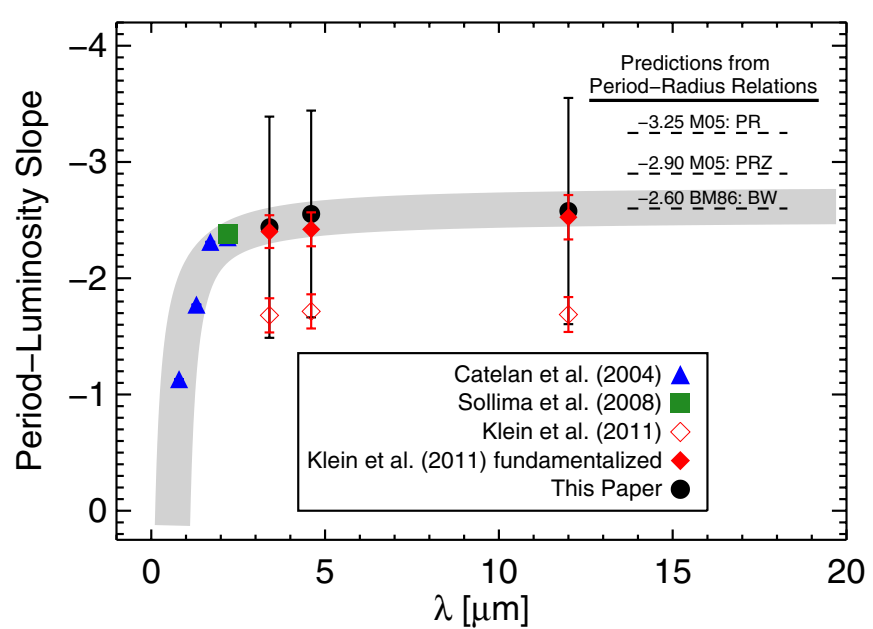

Figure 4. Expected monotonic increase of the slope of the RR Lyrae period-luminosity (PL) relation as a function of increasing wavelength. The asymptotic behavior of the slope, approaching a value of about -2.6 indicates that the PL relation is converging on the period-radius relation, as theory would predict, given that the sensitivity of the surface brightness to temperature rapidly drops as one progressively moves into the infrared. The open diamonds are the slopes published by Klein et al. (2011); the filled (red) diamonds indicate the "fundamentalized" slopes (where we have corrected the periods of the overtone pulsators to their corresponding fundamental periods by adding 0.127 to the log of their observed periods, as in Dall'Ora et al. 2004), based on the data published by Klein et al. (2011) and re-fit for this paper. The optical and near-infrared PL relation slopes are from Catelan et al. (2004), Benedict et al. (2011), and Sollima et al. (2006), while the mid-IR slopes are from this study. The equivalent slopes derived from period-radius relations are from Burki \& Meylan (1986, BM86) and Marconi et al. (2005, M05).

(A color version of this figure is available in the online journal.)

here (e.g., -1.7 compared to our -2.6$)$. This is because in their Bayesian analysis they chose to leave the overtone pulsators in the global solution, without correcting them to their equivalent fundamental periods. We have recomputed the slopes from their data, after applying the appropriate period shift to the overtones, and those PL slopes are plotted in Figure 4. Their slopes and ours now agree well within the errors, but they are still systematically somewhat shallower than our solutions.

\section{THE RUN OF PL SLOPE WITH WAVELENGTH}

For Cepheids it is well known that the slope of the PL relation is a monotonically increasing function of wavelength. In Figure 4 we show that the same overall trend is now made explicit for the first time for the RR Lyrae variables, and for the same physical reasons. As one moves from shorter to longer wavelengths, one is moving from PL relations where the slope is dominated by the trend of decreasing temperature (i.e., decreasing surface brightness) with period, to relations that are dominated by the opposing run of increasing mean radius with period. The plotted slopes of the optical and near-infrared PL relations are representative of a variety of published studies (e.g., Catelan et al. 2004; Benedict et al. 2011; Dall'Ora et al. 2004), while the mid-IR slopes are from this study. As the relative contribution from the temperaturesensitive surface brightness drops off with wavelength, the observed slope is expected to asymptotically approach the wavelength-independent (geometric) slope of the period-area relation. That behavior is indeed seen in Figure 4. Moreover the level at which the plateau is occurring would suggest that the period-radius relation of Burki \& Meylan (1986) (giving a slope of -2.60 , based on Baade-Wesselink studies) 


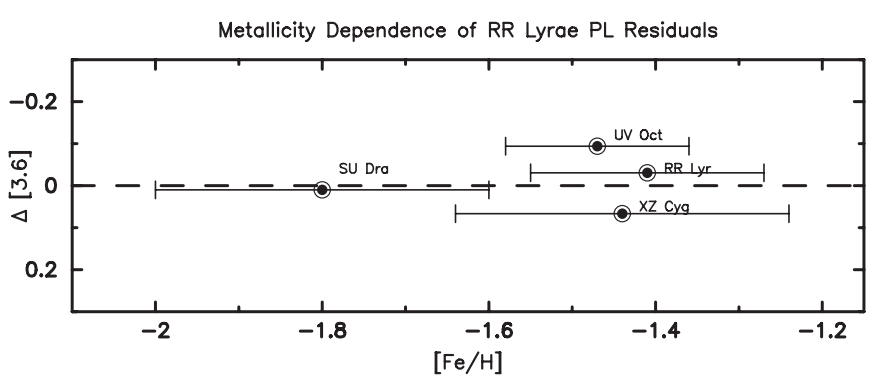

Figure 5. Mid-infrared [W1] $(3.4 \mu \mathrm{m})$ deviations from the mean period-luminosity relation as a function of metallicity. The currently available sample is small, and the metallicity range is limited. No obvious correlation is seen.

is marginally preferred over the period-radius (slope $=-3.25$ ) and period-radius-metallicity (slope $=-2.90$ ) solutions given by Marconi et al. (2005). ${ }^{7}$

From a practical point of view it is not immediately clear what advantage the increased slope of the long-wavelength PL relations would have to offer applications to the distance scale, until it is realized that increased slope in the PL relation is causally and physically connected to decreased width (i.e., decreased intrinsic scatter and therefore increased precision) in the PL relation as shown in the general case by Madore \& Freedman (2012). This effect can be seen for the RR Lyrae variables in Figure 2 of Catelan et al. (2004), and it is apparent here in Figure 3, where the scatter has already reached a minimum in the $K$-band where simultaneously the plateau in slope (seen in Figure 4) is very nearly complete.

\section{A FIRST TEST OF THE METALLICITY DEPENDENCE IN THE MID-IR}

In Figure 5 we plot the measured magnitude residuals from the [W1] $3.4 \mu \mathrm{m}$ PL relation versus the published metallicities of the four RR Lyrae stars in our sample, as given in Table 1 of Benedict et al. (2011). The RR Lyrae stars only sample a 0.4 dex range in $[\mathrm{Fe} / \mathrm{H}]$ so the test is not a strong one, but there is clearly no significant dependence of the already small magnitude residuals on metallicity.

\section{CONCLUSIONS}

As can be seen in the study of Catelan et al. (2004, especially their Figure 2) operating anywhere in the near to mid-IR, from $H=1.6 \mu \mathrm{m}$ (accessible to $H S T$ ) to $3.6 \mu \mathrm{m}$ (accessible to Spitzer now, and with James Webb Space Telescope in the near future) will each accrue the benefits of low scatter and ever decreasing sensitivity (with wavelength) to line-of-sight extinction. Collecting power, availability, and spatial resolution will determine which of these instruments will be used at any given time. But the Population II RR Lyrae variables are proving themselves to be a powerful means of establishing an independent, highly precise, and accurate distance scale that is completely decoupled in its systematics from the Population I Cepheid path to the extragalactic distance scale and the Hubble constant.

This work is based in part on observations made with the Wide-field Infrared Survey Explorer (WISE), which was is operated by the Jet Propulsion Laboratory, California Institute of Technology under a contract with NASA. Support for this work was provided by NASA through an award issued by JPL/Caltech. This research also made use of the NASA/IPAC Extragalactic Database (NED) and the NASA/IPAC Infrared Science Archive (IRSA), both of which are operated by the Jet Propulsion Laboratory, California Institute of Technology, under contract with the National Aeronautics and Space Administration. We thank Fritz Benedict for numerous frank and useful communications. The referee was especially helpful in bringing this paper to a more correct and fruitful completion.

\section{REFERENCES}

Benedict, G. F., McArthur, B. E., Feast, M. W., et al. 2011, AJ, 142, 187 Benedict, G. F., McArthur, B. E., Fredrick, L. W., et al. 2002, AJ, 124, 1695 Burki, G., \& Meylan, G. 1986, A\&A, 159, 261

Cardelli, J. A., Clayton, G. C., \& Mathis, J. S. 1989, ApJ, 345, 245

Catelan, M., Pritzl, B. J., \& Smith, H. A. 2004, ApJS, 154, 633

Cutri, R. M., Wright, E. L., Conrow, T., et al. 2012, WISE All-Sky Release Explanatory Supplement, http://wise2.ipac.caltech.edu/docs/release/allsky/ expsup/

Dall'Ora, M., Storm, J., Bono, G., et al. 2004, ApJ, 610, 269

Fitzpatrick, E. L. 1999, PASP, 111, 63

Freedman, W. L., \& Madore, B. F. 2010, ARA\&A, 48, 673

Freedman, W. L., Madore, B. F., Scowcroft, V., et al. 2012, ApJ, 758, 24

Hanson, R. B. 1979, MNRAS, 186, 875

Indebetouw, R., Mathis, J. S., Babler, B. L., et al. 2005, ApJ, 619, 931

Klein, C. R., Richards, J. W., Butler, N. R., \& Bloom, J. S. 2011, ApJ, 738, 185

Lutz, T. E., \& Kelker, D. H. 1973, PASP, 85, 573

Madore, B. F., \& Freedman, W. L. 2012, ApJ, 744, 132

Marconi, M., Nordgren, T., Bono, G., Schnider, G., \& Caputo, F. 2005, ApJ, 623,133

Marengo, M., Evans, N. R., Barmby, P., et al. 2010, ApJ, 709, 120

McGonegal, R., McAlary, C. W., McLaren, R. A., \& Madore, B. F. 1982, ApJL, 257, L33

Monson, A., Freedman, W. L., Madore, B. F., et al. 2012, ApJ, 759, 146 Persson, S. E., Madore, B. F., Krzeminski, W., et al. 2004, AJ, 128, 2239 Scowcroft, V., Freedman, W. L., Madore, B. F., et al. 2011, ApJ, 743, 76 Sollima, A., Cacciari, C., \& Valenti, E. 2006, MNRAS, 372, 1675

Wright, E. L., Eisenhardt, P. R. M., Mainzer, A. K., et al. 2010, AJ, 140, 1868

Yuan, H. B., Liu, X. W., \& Xiang, M. S. 2013, MNRAS, 430, 2188

\footnotetext{
7 The referee has correctly pointed out that "the period-radius relation provided by Burki \& Meylan (1986) is based on a mix of $\delta$ Scuti, RR Lyrae, and Type II (W Virginis) stars," and that "there is no solid reason why the quoted pulsators should obey the same period-radius relation." At the same time, he/she notes that "the period-radius relation provided by Marconi et al. (2005) is based on a set of RR Lyrae models that cover more than 2 dex in metal abundance. .. and they also account for, at fixed metal abundance, possible evolutionary effects."
} 